\title{
Uncalibrated Trajectory Following with Magnetically Controlled Microrobots*
}

\author{
Jenelle Armstrong Piepmeier, Samara Louise Firebaugh, Caitlin S. Olsen
}

\begin{abstract}
Microrobot systems have demonstrated potential in the areas of manipulation, assembly, and therapeutic tasks at the microscale. This paper investigates uncalibrated visual servoing of microrobot devices in a fluidic environment. Planar actuation is achieved with four electromagnets, and both pointto-point motion and trajectory following are demonstrated. The unique contribution of this paper is the implementation of a dynamic recursive least squares estimation algorithm that is used to control the device as it follows a desired path without any calibration or training steps. Tracking accuracy on the order of 1.6 pixels $(10.5 \mu \mathrm{m})$ is demonstrated for a $200 \mu \mathrm{m}$ diameter microrobot. Initial trials successfully achieved micromanipulation of both 50 and $200 \mu \mathrm{m}$ glass beads.
\end{abstract}

\section{INTRODUCTION}

Tetherless microrobot technology is advancing to new levels and adapting to an increasing number of fields. A challenge facing microrobots is providing power and control. A range of actuation methods, extending from optical [1] to electrostatic [2] and magnetic, have all been demonstrated. An excellent review and comparison of various magnetically actuated microrobot systems can be found in [3], but a selected number of magnetically controlled systems are reviewed here.

Using electromagnetic actuators with ferromagnetic-corebased, Tamaz et al. [4] developed a proportional-integralderivative (PID) controller capable of navigating a $1.5 \mathrm{~mm}$ spherical bead along a predefined path using waypoints. They concluded that an adaptive controller would significantly decrease complications in the system and allow for more robust uses in the biomedical field.

The OctoMag, developed by Kummer et al. [5], is a five degree-of-freedom microrobot controller that has successfully controlled microrobot navigation along preplanned paths while maintaining specific orientations within the workspace. A $2 \mathrm{~mm}$ long microrobot was successfully navigated along a figure-eight path while maintaining its orientation toward the center of the sphere. Another microrobot, $500 \mu \mathrm{m}$ in length, was successfully controlled along a spiral path defined via waypoints, constantly orienting itself toward the vertex of the spiral. In [6] Marino et al. demonstrated an $H_{\infty}$ controller for a linear uncertain dynamical model of the OctoMag for $1 \mathrm{~mm}$ long cylindrical microrobot in low viscosity oil. Tracking accuracies on the order of $270-490 \mu \mathrm{m}$ were achieved.

\footnotetext{
*Support for this project was provided by the Department of the Navy's Program Executive Office for Integrated Warfare Systems (PEO IWS).

J.A. Piepmeier and C. Olsen are with the Systems Engineering Department of the United States Naval Academy, Annapolis, MD 21401

S.L. Firebaugh is with the Electrical and Computer Engineering Department of the United States Naval Academy, Annapolis, MD 21401.
}

Keuning et al. [7] used magnetic actuation to control the path of paramagnetic microparticles with diameters ranging from $60 \mu \mathrm{m}$ to $110 \mu \mathrm{m}$. A proportional-integral (PI) controller was implemented and circular and figure-eight paths were demonstrated. When the microparticle was within 10 pixels of a particular waypoint, it would automatically change course to the next programmed waypoint. Average errors ranged from $4.7 \mu \mathrm{m}$ to $7.0 \mu \mathrm{m}$ with standard deviations on the order of $2.0 \mu \mathrm{m}$.

Belharet et al., [8] used an MRI-based predictive controller to navigate a ferromagnetic robot along a path. With the goal of performing minimally invasive cardiovascular tasks, these microrobots used a linear model to eliminate the need for predefined waypoints. The $250 \mu \mathrm{m}$ microrobot was successful in following these systemgenerated paths. Two dimensional (2D) navigation models with noise resulted in paths following that exhibited a average tracking errors on the order of $230-300 \mu \mathrm{m}$ and 1.2 pixels.

In [9], Pawashe et al. demonstrated model-based learning controllers for micro-object manipulation using side-pushing with a magnetic microrobot operating in a fluid. Modeling included flow velocities induced by the microrobot and equations of motion for the microsphere being manipulated.

All these works required system models and camera calibration. In this paper, we describe a microrobot control scheme that uses uncalibrated visual feedback for an unmodeled system consisting of a microrobot device operating in a fluidic environment observed via a microscope and controlled by four electromagnets. The only a priori information that is required is the number of actuation signals. This controller is developed in Section II.B and demonstrated experimentally in Section IV with both pointto-point motion and path following.

\section{UnCALIBRATED Dynamic Visual SERVo CONTROL}

\section{A. System Model for a Microrobot in a Fluid}

Consider a ferromagnetic microrobot device surrounded by two electromagnet pairs and suspended in between two fluid layers as shown in Fig. 1. The device is subject to a variety of forces including the magnetic control forces and viscous drag. The position of the microrobot is observed in a microscope and measured within the image plane at some position $\vec{x}(t)$.

If the microrobot device is operating in a low Reynolds number regime, then inertial forces are negligible compared to the drag forces as explained in[10]. The Reynolds number is a dimensionless quantity that relates inertial forces to viscous forces in the Navier-Stokes equations for a body moving in an incompressible Newtonian fluid. 
Experimental results presented in [11] and [12] demonstrate a linear relationship between actuation and device velocities for the type of system considered here.

Thus, we assume as system such that the control signal, $\vec{u}(t)$, the state velocity of the microrobot $\dot{\vec{x}}(t)$ as observed in the image plane can be expressed as

$$
\dot{\vec{x}}(t) \cong J(x) \vec{u}(t)
$$

where $J(x)$ is similar to the composite Jacobian matrix used in traditional image based robotic control.

Computing a closed form solution for $J$ is possible, but doing so requires accurate models of the applied magnetic field, the drag coefficients and magnetic properties of the microrobot, the vision system, etc. and initial calibration or training step. Any changes to the physical position of system, components, device geometry, fluid properties, etc. would require a repeated system calibration.

In lieu of careful modeling and calibration, we propose a recursive least squares estimation method for online estimation of the $J$ matrix. Such uncalibrated adaptive methods have been successfully implemented in macro-scale manipulators and mobile robots for a variety of applications with more complex nonlinear system models and higher degrees of freedoms (DOF) [13][14][15].
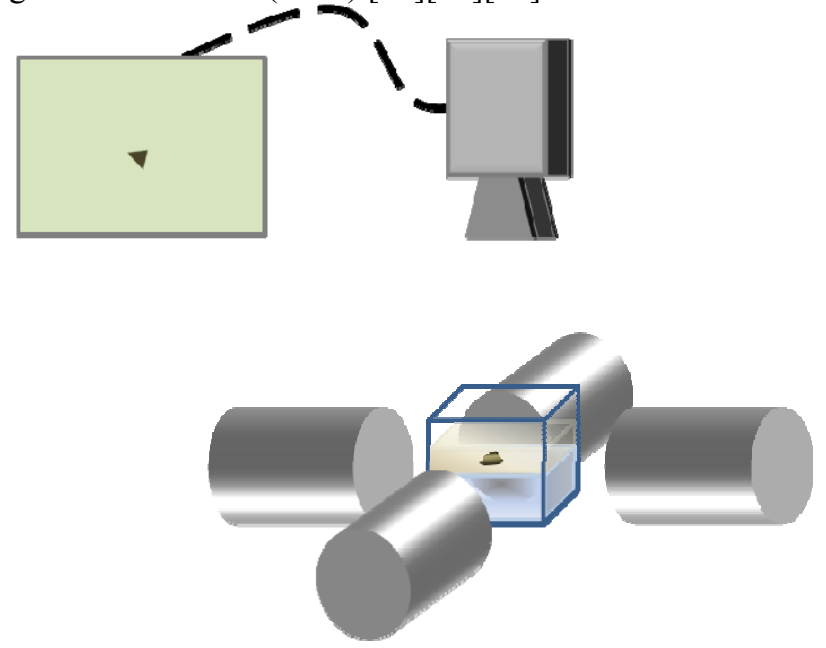

Fig. 1 Microrobot system showing device in fluidic environment with planar control achieved via magnetic control. A microscope and camera system provide image-based feedback.

\section{B. Recursive Least Squares Estimation}

Consider a microrobot system with an observed state $\vec{x}$ that will vary when the control signal $\vec{u}(\mathrm{t})$ is applied to the actuators. The image error between the observed and desired position, $x^{*}(t)$, is given by

$$
f(x)=\vec{x}(t)-\vec{x}^{*}(t)
$$

Let $F(x(t))$ represent the cost function, a function of the image error squared as

$$
F(x(t))=\frac{1}{2} f(x(t))^{T} f(x(t))
$$

Note that $x_{k}^{*}(t)$ may be a constant or a moving target point on a desired motion trajectory. The control algorithm should compute a control signal that will minimize (8) and drive the microrobot to the target point $x_{k}^{*}(t)$.

As shown in [15], a quasi-Newton method utilizing a dynamic Broyden's method will drive the cost function to zero by servoing the robot to following the moving target. The Jacobian estimation scheme uses a rank one update at each iteration.

Let $\Delta$ f represent the change in image error $f_{k}-f_{k-1}$, let $\frac{\partial x_{k}^{*}}{\partial t} h_{t}$ be the change in the desired position, and let $\mathrm{u}_{\mathrm{k}}$ actuation signal consist of the actuation signal for the electromagnets. Then the recursive least squares estimate for the Jacobian $\hat{J}_{k}$ and the desired control signal $\vec{u}_{k}$, is computed at the $k$ th iteration by the following algorithm.

Algorithm 1: Given $f: \in R^{m} ; u_{0}, u_{1} \in R^{n}, \hat{J}_{0} \in R^{m \times n}, P_{0} \in$ $R^{n \times n}, \lambda \in(0,1)$

Do for $k=1,2, \ldots$

$$
\begin{gathered}
\Delta f=f_{k}-f_{k-1} \\
\frac{\partial x_{k}^{*}}{\partial t} h_{t}=\left(x_{k}^{*}-x_{k-1}^{*}\right)\left(t_{k}-t_{k-1}\right) \\
\hat{J}_{k}=\hat{J}_{k-1}+\frac{\left(\Delta f-\hat{J}_{k-1} u+\frac{\partial x_{k}^{*}}{\partial t} h_{t}\right) u^{T} P_{k-1}}{\lambda+u^{T} u} \\
P_{k}=\frac{1}{\lambda}\left(P_{k-1}-\frac{P_{k-1} u_{k} u_{k}^{T} P_{k-1}}{\lambda+u_{k}^{T} P_{k-1} u_{k}}\right) \\
u_{k+1}=-\left(\hat{J}_{k}^{T} \hat{J}_{k}\right)^{-1} \hat{J}_{k}^{T}\left(f_{k}-\frac{\partial x_{k}^{*}}{\partial t} h_{t}\right)
\end{gathered}
$$

End for

End

The matrix $P_{k}$ is the estimate of the covariance matrix of the actuation signal, and $\lambda$ is a weighting factor that effects a memory and reduces the effects of measurement noise on the Jacobian estimation. Values of $\lambda$ closer to 1 effect a longer memory.

Given a non-pathological problem (i.e. a desired path described by of achievable velocities), this algorithm adaptively learns the relationship between the actuation signal and the robot velocities and drives the robot to the desired position. For planar 2DOF position control the image data implies the $m=2$. For an electromagnet array consisting of opposing pairs of magnets, $n=2$. There is no need to calibrate the vision system, compute the viscous drag coefficients, or the magnetic field gradients. Since no system calibration or training is required, the same algorithm will control various microrobot device shapes at arbitrary optical zoom settings.

Such uncalibrated adaptive methods have been successfully implemented in macro-scale manipulators and mobile robots with more complex nonlinear system models and higher degrees of freedoms (DOF) [13][14][15]. 


\section{Practical Implementation}

One final consideration regarding the 2D control signal computed in (13) is necessary for implementation on a physical system. It is possible in the first few steps where the initial magnitude of the control signal may be beyond the physical limitations of the electromagnets. In this event, the signal is scaled such that its magnitude is within the system's capabilities but that the direction is preserved. For a maximum allowable scalar magnitude $u_{\max }$, the scaled actuation signal $\hat{u}_{k+1}$ is used.

$$
\hat{u}_{k+1}=\frac{u_{\max }}{\max \left(u_{k+1}\right)} u_{k+1}
$$

This is similar to the trust region method employed by Jagersand [14] to prevent large motions outside of the estimated model's current area of validity.

While no modeling is necessary for the algorithmic implementation, there are certain practical assumptions that are necessary. It is therefore assumed that the system has been thoughtfully designed such that the magnetic field strength is sufficient to pull the microrobot and overcome viscous drag and other fluid interface reactions.

Furthermore, while a 2DOF system is demonstrated here as described in the following section, the algorithm is extendable to higher degrees of freedom assuming that the size of the measurements vector (denoted by $m$ in the algorithm) is larger than the size of the control vector (denoted as $n$ ). For instance, the measurement vector could consist of position and orientation $(m=3)$ if the control vector included an actuation term that would control the microrobot's orientation.

\section{EXPERIMENTAL SYSTEM}

The uncalibrated control developed in the previous section has been implemented on a system first developed for the Microrobot Challenge held at the 2012 IEEE International Conference on Robotics and Automation (ICRA). A variety of microrobot morphologies (first designed for another application) have been compared in a previous work[11]. The microrobot device used in this work resembles a throwing star, is $20 \mu \mathrm{m}$ thick, and fits within a $200 \mu m$ diameter circle as shown in Fig. 2. The electroplated nickle device was generated by the MEMSCAP MetalMUMPS process ${ }^{1}$.

The microrobot operates at the interface between vegetable oil, and a solution consisting of sodium chloride and sodium bicarbonate dissolved in water, as shown in Fig. 3. The microrobot device will float suspended on fluid B (even without fluid A). This provides a low friction workspace while fluid A (oil) increases the drag forces on the microrobot and results in a more controllable system. The Reynolds number for this system is $\sim 10^{-3}$, therefore the assumption made to ignore inertial terms is valid.

\footnotetext{
${ }^{1} \mathrm{http} / /$ www.memscap.com/products/mumps/metalmumps
}

The robots and fluid are in a $20 \times 20 \times 15 \mathrm{~mm}$ chamber placed at the center of four cylindrical electromagnets arrayed along the four points of the compass. Each magnet is driven with a pulse of amplitude $11 \mathrm{~V}$ and frequency 100 $\mathrm{Hz}$. The duty cycle of the control signal is varied from 0 to $50 \%$. The robots have no permanent magnetization. When placed in a magnetic field they develop an induced magnetization.

A simple actuation scheme with a magnet for each cardinal direction is utilized. Two opposing electromagnets (E-28-150-24 model from Magnetic Sensor Systems) are used to actuate one of the two control signals. By varying the amplitude or duty cycle of square wave input voltages to each electromagnet, the varying magnetic field imposed on the microrobot induces varying forces that propel the microrobot through its workspace.

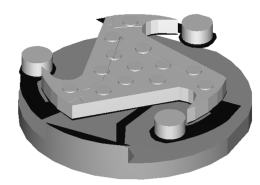

Fig. 2 a) Models of the different microrobot designs derived from the mask layout, rendered in MEMSPro. b) Image of the microrobot as captured by the imaging system used for visual feedback control.

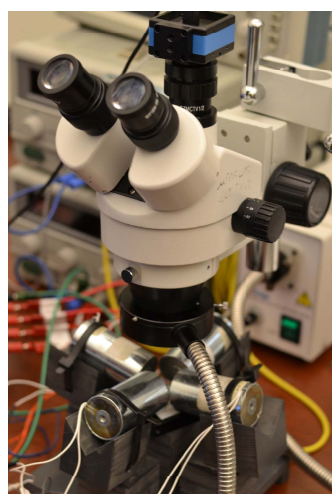

Fig. 3 Experimetnal system showing camera system used for visual feedback and the four electromagnets used for actuation..

One in each pair is used for positive control terms and the other is used for negative terms. At any given time step, only one magnet from each of the two pairs is actuated to pull the robot in the desired direction. Fig. 3 shows the array of electromagnets and the imaging system.

Visual feedback is used to measure the position of the microrobot device using a $740 \times 480$ USB camera. For the experiments presented in the next section, the microscope was set to $20 \times$ with a single pixel corresponding to $6.5 \mu \mathrm{m}$. System integration is achieved in a LabVIEW environment with an $8 \mathrm{~Hz}$ vision update rate. Simple thresholding is used to distinguish the microrobot object from the background, and the centroid of the object is used as the robot position.

The magnets are pulse width modulated with their duty cycle determined by the gain terms adaptively calculated 
within LabVIEW through the algorithm described in equations (4) through (8).

\section{EXPERIMENTAL RESULTS}

\section{A. Static Target}

First, simple point-to-point motion is demonstrated. The algorithm drives the microrobot to the goal position with a velocity term given as $\frac{\partial x_{k}^{*}}{\partial t}=0$. Fig. 4 shows the results for a goal 200 pixels $(1.300 \mathrm{~mm})$ away from the starting point. There is no training or calibration, and $\hat{J}_{0}$ and $P_{0}$ are arbitrarily set equal the identity matrix. The term $\lambda$ is set to 0.99 reducing the effects of system or measurement noise. Fig. 5 demonstrates convergence to the desired position with the error norm in pixels.

The control effort $\hat{u}_{k}$, normalized to a maximum value of 1 , is presented in Fig. 6 with each subplot providing the scaled control signals sent to each electromagnet pair (where a scaled effort of 1 represents a $50 \%$ duty cycle signal sent to the electromagnet). Notice in this figure that even after the microrobot has reached the goal position, the robot may drift prompting a response from the controller to maintain the desired position.

Fig. 7 shows the individual elements of the estimated Jacobian $\hat{J}_{k}$ as they are updated over time. As the robot moves through the workspace, both a sign change and magnitude change are observed. This is not surprising since the initial terms are arbitrary and since the magnetic field varies as the robot moves relative to the electromagnets. Once the robot has achieved the goal position, there is little to no change to the Jacobian.

\section{B. Moving Target}

To demonstrate the ability to follow a desired path or trajectory, the target was prescribed as follows

$$
\vec{x}^{*}(t)=100\left[\begin{array}{l}
\cos (0.035 t) \\
\sin (0.035 t)
\end{array}\right]
$$

This results in an average velocity of 3.5 pixels $/ \mathrm{s}$ or approximately $22.8 \mu \mathrm{m} / \mathrm{s}$. Fig. 8 Shows the trajectory path (superimposed over an image of the robot at the final location) including a portion of the initial approach to the circular path. Once the robot reaches the desired path, it maintains tracking with a steady state error of 1.6 pixels. At this magnification, that corresponds to an average $10.5 \mu \mathrm{m}$ error as shown in Fig. 9.

The scaled control effort is shown in Fig. 10. Compared to the static target case presented in Fig. 6, this control effort varies a great deal as the controller seeks to make small moves keeping the microrobot on the desired path. The target velocity is such that the change in the goal position is on the order of the resolution of the position measurement and the signal is noise dominated. However, the underlying sinusoidal effort is observed with an expected $90^{\circ}$ phase shift between the N/S and E/W electromagnet pairs. The variation in the estimated Jacobian elements can be seen in Fig. 11 demonstrating how the algorithm is essentially adapting the control gains as the robot moves throughout the workspace.

\section{Bead Manipulation}

Potential for small object manipulation led to the development of a trial testing the uncalibrated controller with disturbances. Polystyrene beads with diameters of 50 and $200 \mu \mathrm{m}$ were placed in the workspace of the star shaped microrobot. The beads naturally rest at the interface between the oil and saline solutions, eliminating the need to control the microrobot along the z-axis. Using the manual control system, the star microrobot was capable of pushing and maneuvering both the 50 and $200 \mu \mathrm{m}$ beads. The beads do not stick to the microrobot but instead are dragged primarily by the fluid forces surrounding the microrobot. Because of this, even after leaving the immediate vicinity of the microrobot, the beads continued to travel in the general direction of microrobot motion.

A final experiment was conducted where many $50 \mu \mathrm{m}$ beads were placed in the workspace with the star microrobot. The microrobot was programmed to move in a circle with a 100 pixel radius at a speed of $3 \mathrm{deg} / \mathrm{sec}$. The microrobot ran into a clump of the beads and pushed the beads approximately a quarter of the way along its path. Fig. 12 shows the Jacobian elements as the microrobot encountered the beads. The figure clearly shows a significant change in the Jacobian elements of the controller when the robot encounters the beads. This shows that the uncalibrated controller successfully adjusted for this unforeseen interference.

\section{CONCLUSION}

A vision-based control method is applied to successfully drive a microrobot to a desired target. Both point-to-point motion and path following are demonstrated with a steadystate tracking error of 1.6 pixels $(10.5 \mu \mathrm{m})$. The controller requires no calibration or training phase. This is significant because the same control scheme can be used at different microscopic zoom settings, for different microrobot shapes, and for different viscosity fluids. There is no need for viscous drag modeling or camera calibration which results in a system in which it is extremely easy to test different configurations. This controller was successfully used to push microbeads along a path, highlighting potential for future applications in micromanipulation.

\section{ACKNOWLEDGMENT}

The authors would like to acknowledge the work of J. Raymond, N. Tyson, J. Bradshaw, D. Jobe, B. Stanton, E. Keyes, K. Walsh, and J. Ballman, A.Wessel, P. Dausman, L. Henry, M. Prevatt, S. Johnson, A. Flora, J. Aleshire, K. Boortz, J. Gray, and B. Morris. 


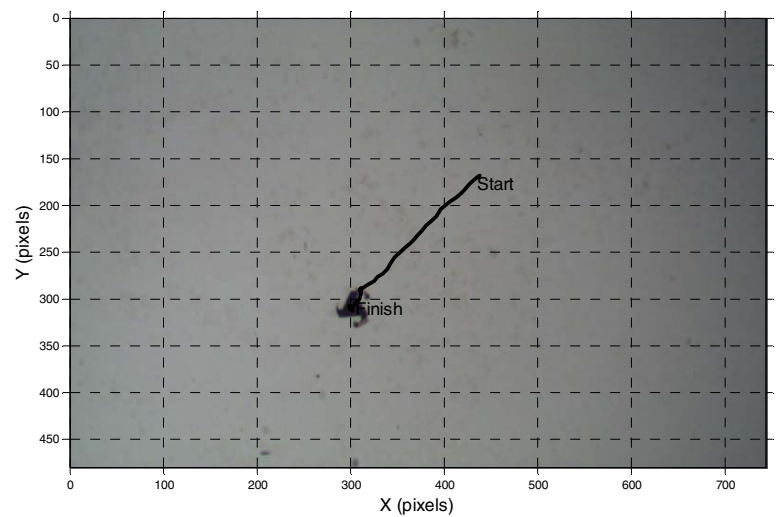

Fig. 4 Point to point motion (static target) with no a priori system modeling or calibration.
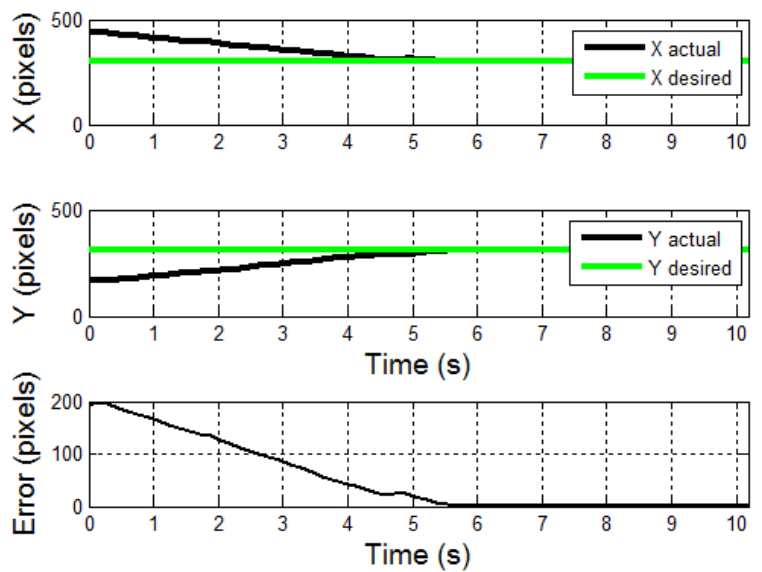

Fig. 5 Robot positions and error norms vs. time showing convergence to the target.
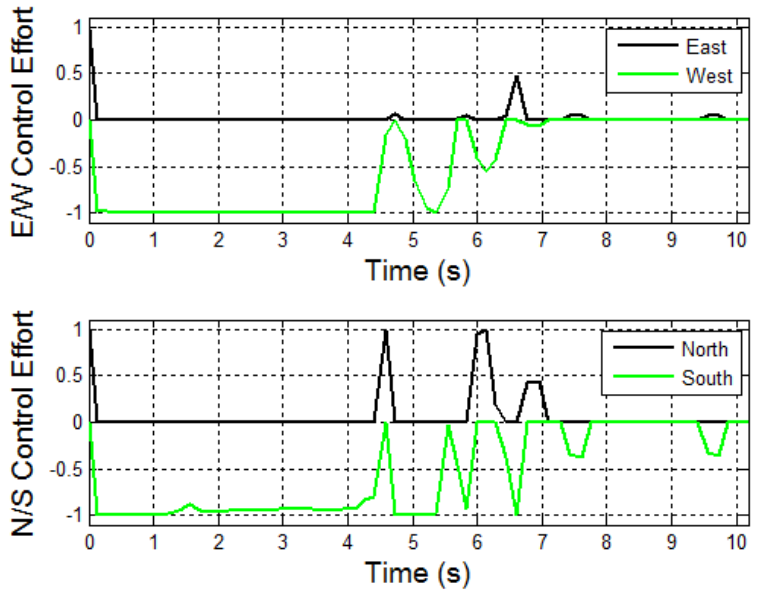

Fig. 6 Normalized control effort showing relative contributions of the N/S and $\mathrm{E} / \mathrm{W}$ magnet pairs. The control effort is limited (as seen in the first $4 \mathrm{~s}$ ) to prevent large motions outside of the field of view.

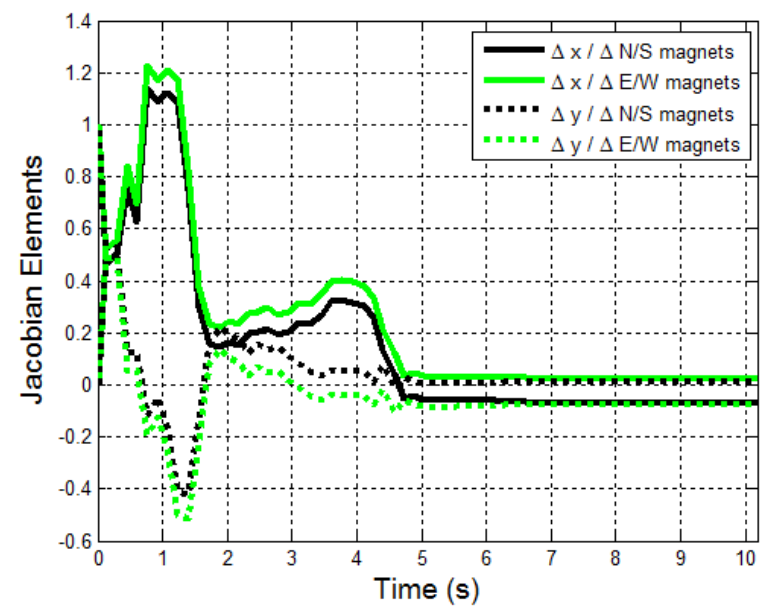

Fig. 7 The four estimated Jacobian elements representing the differential change in the position of the robot in the image plane with respect to differentials in the control inputs.

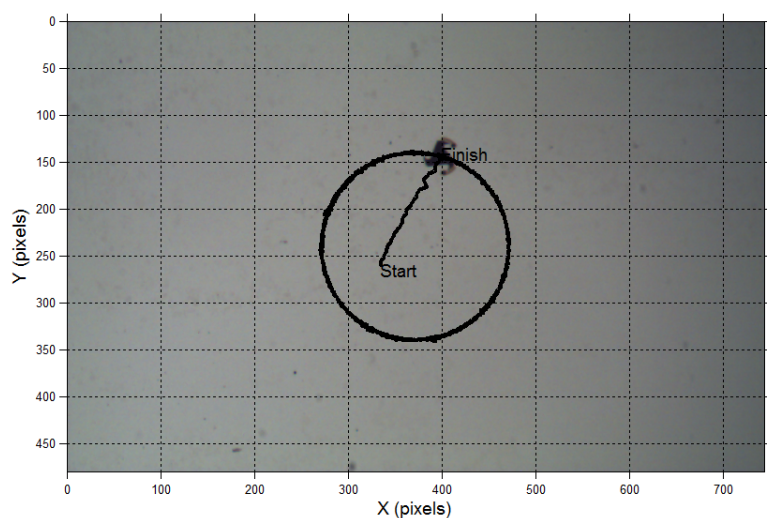

Fig. 8 The microrobot trajectory as it follows a commanded circular path with a $0.65 \mathrm{~mm}$ radius with a speed of approximately $22.8 \mu \mathrm{m} / \mathrm{s}$. Each pixel is $6.5 \mu \mathrm{m}$.

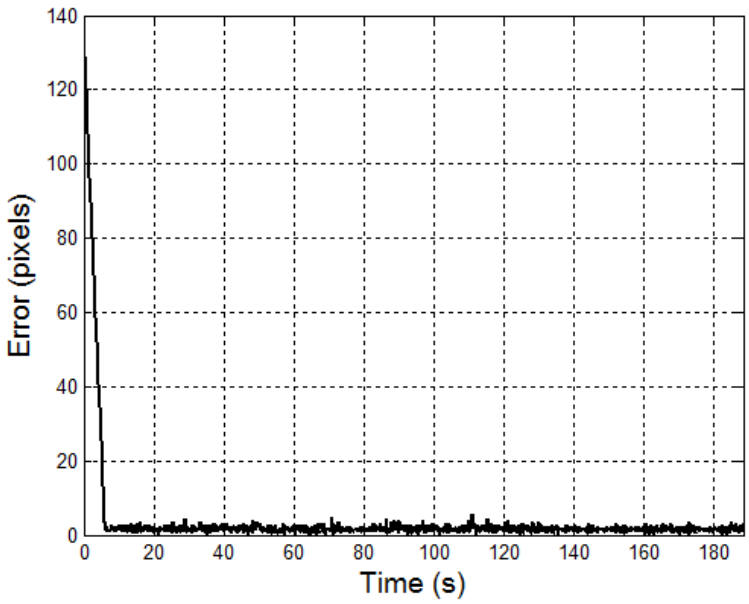

Fig. 9 Error norm between the desired and actual robot position. The steady state error is 1.6 pixels or $10.5 \mu \mathrm{m}$. 

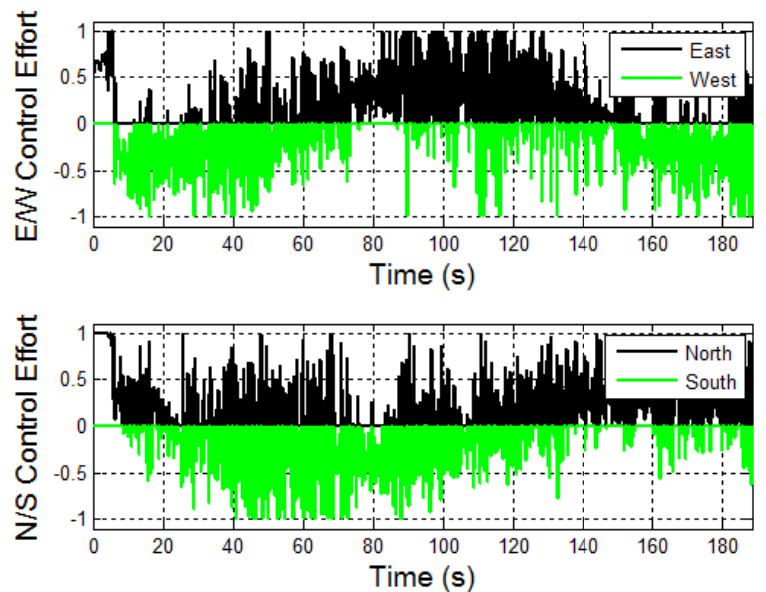

Fig. 10 Normalized control effort showing relative contributions of the N/S and $\mathrm{E} / \mathrm{W}$ magnet pairs. While the control effort is noisy, the underlying sinusoidal variations expected for a circular path are visible.

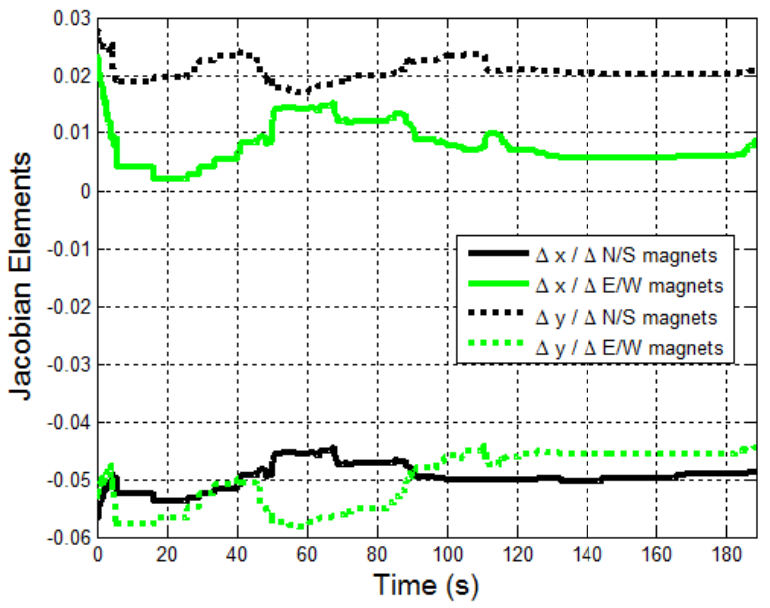

Fig. 11 Changes in the estimated Jacobian elements over time as the microrobot moves through the workspace.

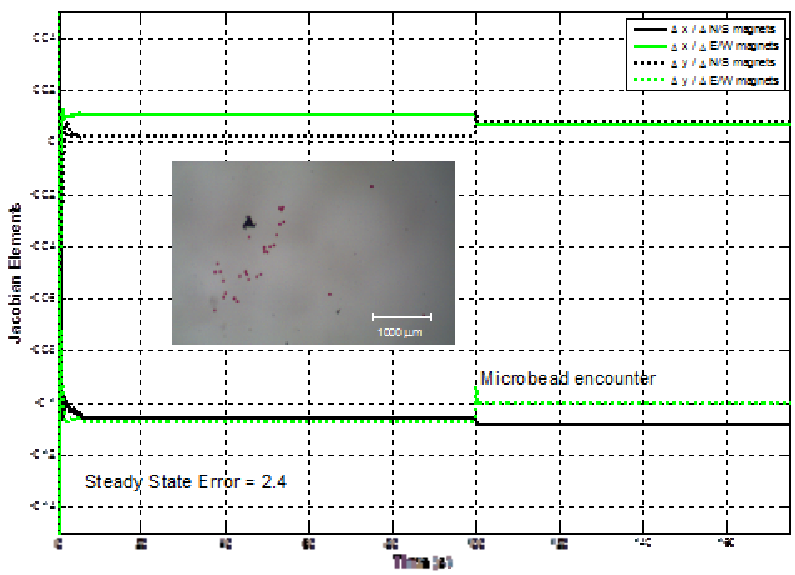

Figure 12: Changes in Jacobian elements as microrobot encounters microbeads.

\section{REFERENCES}

[1] K. Ishii, W. Hu and A. Ohta, "Cooperative Micromanipulation Using Optically Controlled Bubble Microrobots," in IEEE Int. Conf. on
Robotics and Automation, Saint Paul, MN, 2012.

[2] B. Donald, C. Levey, C. McGray, I. Paprotny and D. Rus, "An Untethered, Electrostatic, globally Controllable MEMS Micro-Robot," J. MEMS, vol. 15, no. 1, pp. 1-15, 2006.

[3] S. Bouchebout, A. Bolopion, J.-O. Abrahamians and S. Regnier, "An Overview of Multiple DoF Magnetic Actuated Micro-Robots," $J$. Micro-Nano Mechatronics, vol. 7, no. 4, pp. 97-113, 2012.

[4] S. Tamaz, R. Gourdeau, J. Mathieu and S. Martel, "Real-time MRIbased Control of a Ferromagnetic Core for Endovascular Navigation," IEEE Trans. on Biomedical Engineering, vol. 55, pp. 1854-1863, 2008.

[5] M. Kummer, J. Abbot, B. Kratochvil, R.Borer, A. Sengul and B. Nelson, "OctoMag: An Electromagnetic System for 5-DOF Wireless Micromanipulation," IEEE Trans. on Robotics, vol. 26, no. 6, pp. 1006-1017, 2010.

[6] H. Marino, C. Bergeles and B. Nelson, "Robust H-Infinity Control for Electromagnetic Steering of Microrobots," in IEEE Int. Conf. on Robotics and Automation, Saint Paul, MN, 2012.

[7] J. D. Keunig, J. DeVriessy, L. Abelmanny and S. Misra, "Image-based magnetic control of paramagnetic microparticles in water," in IEEE/RSJ Int. Conf. on Intelligent Robots and Systems (IROS), San Francisco, CA, 2011.

[8] K. Belharet, D. Folio and A. Ferreira, "Endovascular Navigation of a Ferromagnetic Microrobot Using MRI-based Predictive Control," in IEEE/RSJ Int. Conf. on Intelligent Robots and Systems, Taipei, Taiwan, 2010.

[9] C. Pawashe, S. Floyd and M. Sitti, "Two-Dimensional Autonomous Microparticle," IEEE Trans. on Robotics, vol. 28, no. 2, pp. 467-477, 2012.

[10] E. Purcell, "Life at Low Reynolds Number," American J. of Physics, vol. 45, no. 1, pp. 3-11, January 1977.

[11] J. Piepmeier and S. Firebaugh, "Visual Servo Control of Electromagnetic Actuation for a Family of Microrobot Devices," in IEEE Workshop on Robot Vision (WORV), Clearwater, FL, 2013.

[12] M. Sakar, E. Steager, A. Cowley, V. Kumar and G. Pappas, "Wireless Manipulation of Single Cells using Magnetic Microtransporters," in IEEE Int. Conf. on Robotics and Automation (ICRA), Shanghai, China, 2011.

[13] K. Asada and K. Hosoda, "Versatile Visual Servoing Without Knowledge of true Jacobian," in IEEE/RSJ/GI Int. Conf. on Intelligent Robots and Systems, Munich, Germany, 1994.

[14] M. Jagersand, "Visual Servoing Using Trust Region Methods and Estimation of the Full Coupled Visual-Motor Jacobian," in IASTED Applications of Robotics and Control, 1996.

[15] J. A. Piepmeier, G. V. McMurray and H. Lipkin, "Uncalibrated Dynamic Visual Servoing," IEEE Trans. on Robotics and Automation, vol. 20, no. 1, pp. 143-147, 2004. 